\title{
First trimester diagnosis and screening for fetal aneuploidy
}

\author{
Deborah A. Driscoll, $M D^{1}$, and Susan J. Gross, $M D^{2}$; for the Professional Practice and Guidelines Committee
}

\begin{abstract}
Maternal serum screening for neural tube defects and fetal aneuploidy in the second trimester has been incorporated into obstetrical practice over the past two decades. Now, as a result of several multicenter trials, first trimester screening between 11 and 14 weeks has been shown to be an effective and reliable screening test for Down syndrome and trisomy 18. Benefits of first trimester screening include earlier identification of the pregnancy at risk for fetal aneuploidy and anatomic defects, in particular, cardiac anomalies, and the option of earlier diagnosis by chorionic villus sampling, if available. This policy updates the American College of Medical Genetics policy statement entitled Second Trimester Maternal Serum Screening for Fetal Open Neural Tube Defects and Aneuploidy (2004) and complements the sections of American College of Medical Genetic's Standards and Guidelines for Clinical Genetics Laboratories entitled "Prenatal screening for Down syndrome that includes first trimester biochemistry and/or ultrasound measurements." Genet Med 2008:10(1):73-75.
\end{abstract}

Key Words: aneuploidy, multiple marker screening, nuchal translucency

\begin{abstract}
Disclaimer: This guideline is designed primarily as an educational resource for medical geneticists and other health care providers to help them provide quality medical genetic services. Adherence to this guideline does not necessarily assure a successful medical outcome. This guideline should not be considered inclusive of all proper procedures and tests or exclusive of other procedures and tests that are reasonably directed to obtaining the same results. In determining the propriety of any specific procedure or test, the geneticist should apply his or her own professional judgment to the specific clinical circumstances presented by the individual patient or specimen. It may be prudent, however, to document in the patient's record the rationale for any significant deviation from this guideline.
\end{abstract}

Maternal serum screening for neural tube defects and fetal aneuploidy has become part of current obstetrical practice. For over two decades, the focus of such screening programs was based in the second trimester. However, as a result of several multicentered studies conducted in the United States and abroad, first trimester screening between 11 and 14 weeks has been demonstrated to be a useful and reliable option for pregnant women. ${ }^{1-4}$ Benefits of early screening include potentially earlier diagnosis, thereby improving women's reproductive choices, and more accurate pregnancy dating. Although screening for Down syndrome (DS) remains the driving force behind this modality, screening in the first trimester also has the potential to identify other aneuploidies such as Trisomy 18. The addition of ultrasound also allows for the early detection of anatomic defects, particularly cardiac in origin. This current policy updates the American College of Medical Genetics (ACMG) policy statement entitled Second Trimester Maternal Serum Screening for Fetal Open Neural Tube Defects and

From the ${ }^{1}$ University of Pennsylvania Medical Center, Philadelphia, Pennsylvania; and

${ }^{2}$ Albert Einstein College of Medicine, Bronx, New York.

Approved by the Board of Directors, July 28, 2007.

American College of Medical Genetics, 9650 Rockville Pike, Bethesda, MD 20814-3998.

DOI: $10.1097 /$ GIM.0b013e31815efde8
Aneuploidy (2004) and complements the sections of ACMG's Standards and Guidelines for Clinical Genetics Laboratories entitled "Prenatal screening for Down syndrome that includes first trimester biochemistry and/or ultrasound measurements."

\section{DIAGNOSIS OF FETAL ANEUPLOIDY}

Although there is exciting research and innovation in the field of noninvasive testing for fetal aneuploidy, at present there are two tests, both invasive, which are used in a routine manner to determine the presence of fetal aneuploidychorionic villus sampling (CVS) and amniocentesis. CVS is performed in the first trimester from 10 through 13 weeks' gestation, whereas amniocentesis can be performed starting at 15 weeks' gestation. More recent literature suggests that the risk of pregnancy loss from sonographically directed amniocentesis is 1 in $300-600,{ }^{5,6}$ with one study suggesting that there may be no significant increase over the background miscarriage risk. ${ }^{7}$ Likewise, the risk of pregnancy loss with CVS is also low, particularly for transabdominal procedures. ${ }^{8}$ Transcervical CVS loss rates are slightly increased. Based on the relative safety of these diagnostic procedures, and in deference to patient preference and autonomy, the American College of Ob- 
stetricians and Gynecologists recently recommended that all pregnant women regardless of maternal age should have the option of having an invasive diagnostic test to definitively identify not only DS, but all major fetal aneuploidies. Such diagnostic testing should be made available if requested after appropriate counseling including risks and benefits. Likewise, women who do not want any further information regarding chromosomal status of their fetus should not be required to undergo any further testing or screening. Screening provides such an option for those women who would like to further refine their risk before deciding whether or not to undergo invasive testing.

\section{FIRST TRIMESTER SCREENING}

\section{Serum markers}

Presently, the two markers currently in clinical practice with demonstrated utility are pregnancy-associated plasma protein A (PAPP-A), which is typically reduced and human chorionic gonadotrophin (hCG), which is elevated in DS. The overall data suggest that free beta hCG is likely superior. ${ }^{9}$ However, access to free beta hCG has been limited and, therefore, many programs in the United States use intact hCG.

\section{Ultrasound}

The big breakthrough in first trimester screening was the advent of the nuchal translucency (NT) measurement. Between 11 and 14 weeks, a clearly demarcated fluid-filled space can be seen behind the fetal neck. This space is present in all fetuses. An increased NT measurement is significantly associated with fetal DS and other aneuploidies. The detection rate for DS is approximately $70 \%$ with a $5 \%$ false-positive rate. Other sonographic findings are being investigated as potential markers for DS. Absence of the nasal bone is associated with DS but its value as a screening test in the general population is controversial. As with the NT measurement, standardization of the technique and quality assessment programs are essential. ${ }^{10}$ Some programs have incorporated nasal bone assessment into their first trimester screening protocol. ${ }^{11}$ Now there is also substantial evidence that congenital heart defects may present with increased NT measurement. ${ }^{12}$ Other findings associated with increased NT measurements include diaphragmatic hernias, skeletal dysplasias, and a variety of genetic syndromes. ${ }^{10}$ Based on the current scientific evidence, the American College of Obstetricians and Gynecologists recommends that patients with a fetal NT measurement of $3.5 \mathrm{~mm}$ or higher be offered a targeted ultrasound, echocardiogram, or both.

\section{Combined ultrasound and serum markers}

Multiple studies have demonstrated that the most effective DS screening approach in the first trimester is the combination of maternal age, NT measurement, and serum markers. The DS detection rates in four major studies range from 79 to $90 \%$ with a $5 \%$ false-positive rate. A consistent finding across all studies is the necessity for standardization and rigorous quality control both in the laboratory assays and NT measurements. Combined algorithms are also available for Trisomy $18 .{ }^{13}$ It is acknowledged that combined ultrasound and serum screening for multifetal gestations remains less sensitive than in singleton pregnancies. There are insufficient prospective studies to determine actual detection rates so that laboratories that do provide risk results are using mathematical models. There is also the ongoing concern that serum marker levels reflective of both pregnancies are actually averaged, which is a problem across all trimesters. NT measurement alone is useful in screening multiple gestations for DS but is associated with a higher positive screening rate.

\section{SCREENING IN BOTH FIRST AND SECOND TRIMESTERS}

There have been several different approaches to improve screening rates based on combining first and second trimester screening. These include integrated testing, where NT measurement and PAPP-A in the first trimester are combined with the second trimester screening that includes alphafetoprotein, hCG, unconjugated estriol, and dimeric inhibin-A; however, with this approach results are not available until the second trimester. ${ }^{14}$ Sequential screening takes advantage of the higher detection rates achieved with an integrated approach but discloses the first trimester results, which allows a patient the option of CVS and earlier termination in the event of an affected fetus. Independent assessment of DS risk by both first and second trimester screening is discouraged because of the high false-positive rates associated with this approach. ${ }^{15}$ Most recently, contingency screening has been proposed whereby only those women with a result in a predefined intermediate range undergo testing in the second trimester. ${ }^{16}$ These various combinations offer detection rates of over $90 \%$ with reduced falsepositive rates.

\section{RECOMMENDATIONS}

The American College of Medical Genetics recommends the following:

- All women should have the option of invasive diagnostic testing for fetal aneuploidy by CVS, if available, or amniocentesis. Benefits include diagnosis of all major aneuploidies and large chromosomal rearrangements.

- For women who do not want any information regarding fetal aneuploidy status, following appropriate documentation, no other testing or screening is required.

- First trimester screening (NT, PAPP-A, and hCG) is an acceptable, cost effective approach for DS risk screening for women if they present early in pregnancy (before 14 weeks' gestation).

- Incorporation of the nasal bone assessment into the first trimester screening protocol is optional and should be limited to clinicians who have specific training and participate in an ongoing quality assurance program. 
- Women presenting in the second trimester should be offered multiple marker screening as described in the ACMG policy statement entitled Second Trimester Maternal Serum Screening for Fetal Open Neural Tube Defects and Aneuploidy (2004) (www.acmg.net).

- Approaches that use first and second trimester screening have improved detection rates and lower false-positive rates. However, providers and centers must be prepared to ensure that there is adequate follow-up and nondisclosure is acceptable to the patient if they choose to offer integrated screening. Sequential or contingency screening with disclosure of the first trimester results may be preferable for some providers and patients.

- Women should be informed of the adjusted risk for DS and allowed to make decisions based on this number, because individuals will weigh risk/benefits differently and are capable of such decision-making. Because age is no longer used as a cutoff to offer invasive testing, it is no longer logical to use predetermined cutoffs based on age risk for screening programs.

- First trimester screening can be used in multifetal pregnancies; however, women should be made aware of the limitations of screening in this setting.

- An NT measurement of $3.5 \mathrm{~mm}$ or greater, even in the setting of a low risk for aneuploidy screen result or normal fetal karyotype should prompt a detailed anatomic ultrasound, echocardiogram, or both.

- Women who have elected to have first trimester screening and/or CVS should be offered MSAFP screening and/or an anatomic survey, optimally between 16 and 20 weeks' gestation for the detection of neural tube defects.

- First trimester screening requires adherence to strict standards and maintenance of quality, both in the laboratory and ultrasound units. Sonographers must be appropri- ately trained in the proper technique of NT measurement and have appropriate certification through available organizations.

\section{References}

1. ACOG Committee on Practice Bulletins. ACOG Practice Bulletin No. 77: screening for fetal chromosomal abnormalities. Obstet Gynecol 2007;109:217-227.

2. Malone FD, Canick JA, Ball RH, Nyberg DA, et al. First-trimester or second-trimester screening, or both, for Down's syndrome. N Engl J Med 2005;353:2001-2011.

3. Spencer K, Souter V, Tul N, Snijders R, et al. A screening program for trisomy 21 at 10-14 weeks using fetal nuchal translucency, maternal serum free beta-human chorionic gonadotropin and pregnancy-associated plasma protein-A. Ultrasound $\mathrm{Ob}$ stet Gynecol 1999;13:231-237.

4. Wald NJ, Rodeck C, Hackshaw AK, Rudnicka A. SURUSS in perspective. BJOG 2004;111:521-531.

5. Caughey AB, Hopkins LM, Norton ME. Chorionic villus sampling compared with amniocentesis and the difference in the rate of pregnancy loss. Obstet Gynecol 2006; 108:612-616.

6. Scott F, Peters H, Boogert T, Robertson R, et al. The loss rates for invasive prenatal testing in a specialised obstetric ultrasound practice. Aust NZJ Obstet Gynecol 2002; 42:55-58.

7. Eddleman KA, Malone FD, Sullivan L, Dukes K, et al. Pregnancy loss rates after midtrimester amniocentesis. Obstet Gynecol 2006;108:1067-1072.

8. Alfirevic Z, Sundberg K, Brigham S. Amniocentesis and chorionic villus sampling for prenatal diagnosis. Cochrane Database Syst Rev. 2003;(3):CD003252.

9. Spencer K. Aneuploidy screening in the first trimester. Am J Med Genet C Semin Med Genet 2007;145:18-32.

10. Sonek J. First trimester ultrasonography in screening and detection of fetal anomalies. Am J Med Genet C Semin Med Genet 2007;145:45-61.

11. Cicero S, Avgidou K, Rembouskos G, Kagan KO, et al. Nasal bone in first-trimester screening for trisomy 21. Am J Obstet Gynecol 2006;195:109-114.

12. Makrydimas G, Sotiriadis A, Huggon IC, Simpson J, et al. Nuchal translucency and fetal cardiac defects: a pooled analysis of major fetal echocardiography centers. Am J Obstet Gynecol 2005;192(1):89-95.

13. Wapner R, Thom E, Simpson JL, Pergament E, et al. First-trimester screening for trisomies 21 and 18. N Engl J Med 2003;349:1405-1413.

14. Sharma G, McCullough LB, Chervenak FA. Ethical considerations of early (first vs. second trimester) risk assessment disclosure for trisomy 21 and patient choice in screening versus diagnostic testing. Am J Med Genet C Semin Med Genet 2007;145: 99-104.

15. Platt LD, Greene N, Johnson A, Zachary J, et al. Sequential pathways of testing after first-trimester screening for trisomy 21. Obstet Gynecol 2004;104:661-666.

16. Benn P, Wright D, Cuckle H. Practical strategies in contingent sequential screening for Down syndrome. Prenat Diagn 2005;25:645-652. 